\title{
空气湿度对人员可接受热环境的影响及评价
}

\author{
杜晨秋, 李百战 ${ }^{*}$, 刘红, 李超 \\ 重庆大学绿色建筑与人居环境营造国际合作联合实验室, 重庆 400045 \\ *联系人, E-mail: baizhanli@cqu.edu.cn \\ 2019-08-30 收稿, 2019-09-29 修回, 2019-10-08 接受, 2019-12-13 网络版发表 \\ 国家自然科学基金(51908079)和国家重点研发计划(2016YFC0700300, 2017YFC0702704)资助
}

\begin{abstract}
摘要 室内温湿度对人员舒适健康有着显著影响, 尤其在偏热和偏冷环境下. 如何评价较大空气湿度范围内温湿 度耦合作用及其影响尚缺乏系统认识。我们在人工气候室分别开展了冬夏季涵盖偏冷到偏热 $\left(16 \sim 32^{\circ} \mathrm{C}\right)$ 、低湿到 高湿(15\% $85 \%)$ 共 40 个工况的人体热舒适实验，累计 800 余人次. 结果显示，相对湿度对人员热舒适的影响随温度 升高逐渐显著 $\left(\right.$ 夏季 $32^{\circ} \mathrm{C} /$ 冬季 $28^{\circ} \mathrm{C}$ ), 湿感觉随相对湿度增加而显著增加; 低温下空气绝对含湿量低, 人体各部位刺 激感比例随相对湿度降低而增加; 低温下人员感知空气品质随暴露时间增加而降低, 高温下人员适应性提高了人 员对空气品质感知. 研究计算了不同工况下人体呼吸总热损失, 并建立其与人员热感觉(thermal sensation vote, TSV)和可感知空气品质(air quality vote, AQV) 的关系：随着呼吸总损失增加，人员热感觉线性降低(回归系数: -0.66 ), 感知空气品质升高(回归系数: 0.2). 基于 $\mathrm{TSV} \in(-0.5,+0.5)$ 和 $\mathrm{TSV} \in(-0.75,+0.75)$, 得到了冬夏季满足 $90 \%$ 和 $80 \%$ 人员满意率的标准有效温度(standard effective temperature, SET)上下限值, 和等SET条件下温度-相对湿度 $(30 \%$ 70\%)舒适区间，且大于国际标准ASHRAE 55推荐的温度区间。研究量化了空气湿度对人员热感觉的影响，揭示了 低湿和高湿通过影响皮肤表面散热和呼吸蒸发热损失影响人员可接受温度区间, 研究结果可为提出合理室内空气 湿度设计参数、保障室内热环境舒适健康营造提供一定参考.
\end{abstract}

关键词空气温度, 相对湿度, 热舒适, 空气品质, 温湿度区间

空气湿度作为热环境设计和评价的重要物理参数, 由于不同于温度感知, 至今尚未有研究发现人体存在 特定的响应湿度信号的感受器来感受湿度刺激并引起 调节响应 ${ }^{[1]}$. 因此, 关于湿度对人体舒适健康的影响机 理, 以及适宜的热环境设计, 一直是建筑环境领域学者 的研究热点.

大量研究已经证实，中性热环境下空气湿度对人 体热舒适影响较小, 加上适应后的人员对湿度不敏 感 ${ }^{[2]}$, 因而较大温度范围内湿度对人体热舒适的影响 可以忽略 ${ }^{[3,4]}$. 但当环境温度升高, 或者人体代谢加强 时 $^{[5]}$, 高湿度会抑制人体出汗, 减小蒸发热损失, 从而
增加人体热感觉, 降低可接受的温度上限 ${ }^{[6,7]}$, 且湿度越 高, 可接受温度的带宽越窄 ${ }^{[8]}$. 更重要的是, 随着建筑围 护结构性能提高、密闭性增加以及通风率降低, 较高 的空气湿度还会引起普遍的建筑室内潮湿问题 ${ }^{[9]}$, 诱 发一系列的潜在危险因素，包括增加建筑墙体或材料 表面潮湿程度、促进室内尘螨和霉菌等微生物生长、 诱发室内装修材料中各种化学污染物挥发等, 影响室 内空气品质, 使人群呼吸系统相关疾病患病风险增 加 $^{[10]}$. 一些在欧洲、北美、亚洲等地区开展的大样本 横断面调研结果显示 ${ }^{[11 ~ 15]}$, 室内潮湿及其表征(霉点湿 点、窗户凝水、水损、发雼气味等) 与儿童哮喘、鼻

引用格式: 杜晨秋, 李百战, 刘红, 等. 空气湿度对人员可接受热环境的影响及评价. 科学通报, 2020, 65: 311-324

Du C Q, Li B Z, Liu H, et al. Effect of air humidity on human acceptable thermal environments and evaluation (in Chinese). Chin Sci Bull, 2020, 65: 311 324, doi: 10.1360/TB-2019-0461 
炎及过敏性症状(喘息、喉咙痛、流鼻涕)之间有着显 著的相关性，是儿童哮喘、鼻炎等症状出现的显著危 险因素，更进一步反映了高空气湿度对人员舒适健康 的影响.

Wei等人 ${ }^{[16]}$ 分析了评价室内空气品质的 6 个关键指 标, 指出相对湿度是影响室内空气品质的关键因素. 空 气湿度的变化会直接影响呼吸道吸人空气的冷却效 率 $^{[17]}$, 低温低湿的空气会增加呼吸道和外界的对流换 热和蒸发换热, 而高温湿度情况下人体的呼吸秥膜冷 却效果降低，人员可感知室内空气品质下降. Fang等 人 $^{[18,19]}$ 通过实验发现，温度不变、湿度增加和湿度不 变、温度增加条件下，人员的感知空气品质不满意率 均显著增加. 相反, 干燥的空气会影响人体黏膜的生理 特性, 较低的空气水分含量会加速泪液蒸发速度, 尤其 是人体角膜前泪液膜 ${ }^{[20]}$, 造成人员眨眼频率降低, 引起 眼部不适等症状. Fanger ${ }^{[21]}$ 曾指出, 短期暴露情况下人 员对干冷空气的可接受空气品质更好, 但长期暴露情 况下, 相对湿度大于 $40 \%$ 比低于 $30 \%$ 的环境对眼睛和上 呼吸道更好 ${ }^{[22]}$.

但是, 相对湿度对人体舒适健康的影响与温度存 在耦合关系. Nevins等人 ${ }^{[23]}$ 通过偏冷-中性-偏热环境中 热舒适实验, 发现相对湿度降低 $10 \%$, 温度可相应增加 $0.3^{\circ} \mathrm{C}$. 从人体热平衡的角度来看, 除了极端条件, 湿度 对人体整体热损失的影响较小，人员的热感觉、皮肤 温度等基本不受湿度影响 ${ }^{[24,25]}$, 因此空调系统可以在 较大的湿度范围内考虑温度设置而不考虑湿度作用. 早期的美国标准ASHRAE 55给出的夏季湿球温度不高 于 $20^{\circ} \mathrm{C}$ 、冬季不高于 $18^{\circ} \mathrm{C}$, 参考的是保证人体皮肤表 面的湿润度维持在 0.2 左右, 但这一结果并没有科学的 实验研究背景 ${ }^{[26]}$. 现行最新版ASHRAE $552017^{[27]}$ 给 出的湿度上限 $0.012 \mathrm{~kg} / \mathrm{kg}$ 考虑的也是湿度增加可能引 起墙面结露、材料腐蚀, 以及尘螨、真菌和其他微生 物生长的风险增加, 而不是湿度对热舒适影响. 国际标 准ISO7730-2005 ${ }^{[28]}$ 也并未对湿度做出规定, 而是认为 相对湿度每增加 $10 \%$, 等价于空气温度升高 $0.3^{\circ} \mathrm{C}$. 其他 一些关于热舒适和建筑节能的相关标准, 如美国ASH-

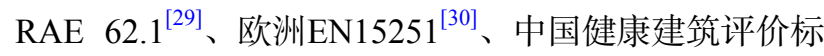
准T/ASC 02-2016 $6^{[31]}$ 等, 出于通风、节能等考虑, 对湿 度设计也给出了一定的参考范围，但其适用性还有待 进一步研究.

综上，一般热环境范围内空气湿度的作用并不明 显, 因此对于适宜的温湿度范围, 研究并未得出一致的
结果 ${ }^{[32]}$. 大量关于湿度对空气品质影响的研究表明, 要 营造一个舒适健康的人居环境，其温湿度设计不仅需 要基于人员的热舒适评价，也应考虑湿度耦合温度对 人员感知空气品质和健康的影响. 而室内热环境设计 要实现这一目的, 应首先明晰温湿度的相互作用对人 员热舒适的影响，并基于人员评价和可接受的合理温 湿度区间, 才能实现合理地调节室内温湿度和空调系 统的节能运行. 基于此, 本研究利用人工气候室在冬夏 季营造较大范围内的湿度变化，开展不同温湿度水平 下人员热舒适评价的基础研究，旨在全面认识人员在 低湿到高湿较大范围内的主观热反应评价, 揭示湿度 与温度的耦合作用，提出综合考虑人员热感觉和感知 空气品质的适宜的温湿度区间，以期更全面地评价空 气湿度对室内温度设计和调控的影响, 为室内热环境 的舒适健康营造及建筑节能提供一定基础参考和 支撑.

\section{1 研究方法}

\section{1 实验平台}

实验在重庆大学人工气候室(尺寸 $4 \mathrm{~m}($ 长) $\times 3 \mathrm{~m}$ (宽) $\times 3 \mathrm{~m}$ (高))开展. 气候室采用内围护结构, 可精确控 制室内热环境参数: 干球温度控制范围 $-5 \sim 40^{\circ} \mathrm{C}$, 精度 $\pm 0.3^{\circ} \mathrm{C}\left(10^{\circ} \mathrm{C}\right.$ 以下, $\left.\pm 0.5^{\circ} \mathrm{C}\right)$; 相对湿度(relative humidity, RH)控制范围 $10 \%$ 90\%, 精度 $\pm 5 \%$. 其中, 气候室低湿环 境由转轮除湿机控制，相对湿度控制范围为 $0 \sim 100 \%$, 控制精度 $\pm 2 \%$. 实验中, 气候室空气经处理后由顶部孔 板送人室内, 通过底部孔板回风, 整个工作区气流均匀, 且控制工作区风速 $\leqslant 0.1 \mathrm{~m} / \mathrm{s}$, 近似静风环境. 需要说明 的是, 由于人工气候的室外环境为房间的室内环境, 且 采用双层结构设计, 围护结构采用双面涂塑钢板和 $100 \mathrm{~mm}$ 聚氨酯发泡填充, 不受室外环境变化和太阳辐 射的影响. 另有一控制室与气候室相邻, 通过变频空调 控制维持中性热环境, 实验中做准备室用. 人工气候室 内外环境和实验现场测试示意如图1.

\section{2 受试者}

本实验采用自愿方式招募受试者. 受试者均为重 庆大学在校学生, 每一季度实验男女受试者各招募 10 名, 减小性别差异影响. 要求受试者在重庆居住 1 年以 上，身体状况良好，且实验前 1 天禁止剧烈运动或饮酒 等. 受试者基本信息见表1. 实验时受试者服装由实验 


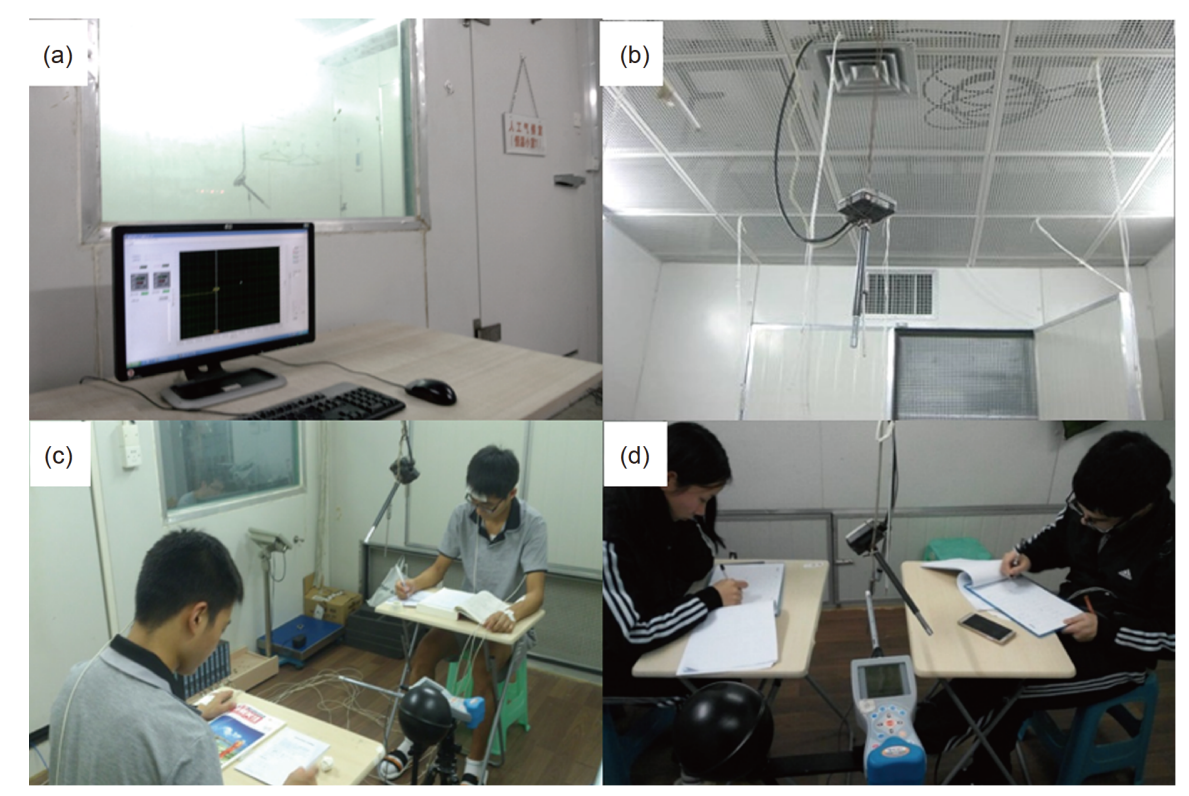

图 1 (网络版彩色)人工气候室和实验示意图. (a) 气候室外; (b) 气候室内; (c, d) 现场实验

Figure 1 (Color online) Artificial climate chamber and experiment. (a) Outside of climate chamber; (b) inside of climate chamber; (c, d) on-site tests

表 1 受试者基本信息参数(平均值土标准差)

Table 1 Detailed information of subjects (mean \pm SD)

\begin{tabular}{|c|c|c|c|c|c|}
\hline 季节 & 性别 & 人数 & 年龄(岁) & 身高(cm) & 体重 $(\mathrm{kg})$ \\
\hline \multirow{2}{*}{ 夏季 } & 男 & 10 & $22.8 \pm 1.5$ & $172.6 \pm 4.5$ & $70.3 \pm 9.3$ \\
\hline & 女 & 10 & $23.1 \pm 1.0$ & $161.0 \pm 4.9$ & $49.6 \pm 4.5$ \\
\hline 总计 & & 20 & $23.0 \pm 1.2$ & $166.8 \pm 4.7$ & $59.9 \pm 7.5$ \\
\hline \multirow{2}{*}{ 冬季 } & 男 & 10 & $22.2 \pm 1.0$ & $176.0 \pm 4.7$ & $65.6 \pm 8.5$ \\
\hline & 女 & 10 & $22.0 \pm 0.7$ & $161.3 \pm 6.7$ & $49.5 \pm 6.5$ \\
\hline 总计 & & 20 & $22.1 \pm 0.1$ & $168.5 \pm 7.4$ & $57.6 \pm 8.0$ \\
\hline
\end{tabular}

人员统一提供, 夏季为棉质短袖 + 薄长裤 + 薄棉袜 + 休闲 鞋, 整体服装热阻约 $0.4 \mathrm{clo}\left(1 \mathrm{clo}=0.155 \mathrm{~m}^{2}{ }^{\circ} \mathrm{C} / \mathrm{W}\right)^{[27]}$, 冬 季为棉质秋衣 + 毛衣 + 棉服 $($ 外套 $)+$ 棉质秋裤 + 厚外裤 + 运动鞋, 整体服装热阻约 1.2 clo.

\section{3 工况设计}

实验主要研究不同湿度水平对人员热反应的影响, 而湿度和温度的耦合作用不能忽略 ${ }^{[25]}$. 因此, 结合重庆 地区全年室内温度波动范围, 实验分别选择在冬季和 夏季开展，均对应设计了偏凉、中性、偏暖、热 4 种温 度水平, 每一温度工况对应低湿到高湿 5 种相对湿度水 平, 共 40 个实验工况, 具体见表 2 . 为避免受试者个体差 异对实验结果的影响, 实验均采用被试内设计, 要求冬
季招募的 20 名受试者参加冬季的所有 20 个工况实验, 夏季招募的 20 名受试者参加夏季的所有 20 个工况实验 (表2). 冬夏季实验最终累计开展 800 人次，可确保实验 结果的有效性和数据可信度.

Gagge等人 ${ }^{[33]}$ 基于二节点模型, 提出了包含空气温 度、相对湿度、风速、辐射温度等热环境参数以及服 装热阻和活动水平的综合热舒适指标一一标准有效温 度(standard effective temperature, SET), 即相对湿度为 $50 \%$ 的假想等温环境下, 人体着装对应活动水平的标准 服装时，与真实环境有相同的皮肤温度和皮肤湿润率， 则该环境温度即为标准有效温度 ${ }^{[27]}$. 表2同时给出了相 应设计工况计算得到的SET值. 可以看出, SET随温度 的增加迅速增加，同一温度下随相对湿度增加而增加， 
表 2 冬夏季实验工况 ${ }^{\text {a) }}$

Table 2 Experimental conditions in summer and winter

\begin{tabular}{|c|c|c|c|c|c|c|c|c|c|}
\hline \multicolumn{5}{|c|}{ 夏季设计工况 } & \multicolumn{5}{|c|}{ 冬季设计工况 } \\
\hline 温度 $\left({ }^{\circ} \mathrm{C}\right)$ & $\mathrm{RH}(\%)$ & 风速 & 服装热阻 & $\operatorname{SET}\left({ }^{\circ} \mathrm{C}\right)$ & 温度 $\left({ }^{\circ} \mathrm{C}\right)$ & $\mathrm{RH}(\%)$ & 风速 & 服装热阻 & $\operatorname{SET}\left({ }^{\circ} \mathrm{C}\right)$ \\
\hline \multirow[t]{5}{*}{23} & 15 & $\leqslant 0.1 \mathrm{~m} / \mathrm{s}$ & 0.4 clo & 20.7 & 16 & 15 & $\leqslant 0.1 \mathrm{~m} / \mathrm{s}$ & 1.2 clo & 20.8 \\
\hline & 30 & & & 20.9 & & 30 & & & 20.9 \\
\hline & 50 & & & 21.1 & & 50 & & & 21.1 \\
\hline & 70 & & & 21.4 & & 70 & & & 21.2 \\
\hline & 85 & & & 21.6 & & 85 & & & 21.3 \\
\hline \multirow[t]{5}{*}{26} & 15 & & & 23.9 & 20 & 15 & & & 24.2 \\
\hline & 30 & & & 24.0 & & 30 & & & 24.4 \\
\hline & 50 & & & 24.4 & & 50 & & & 24.7 \\
\hline & 70 & & & 24.9 & & 70 & & & 25.2 \\
\hline & 85 & & & 25.5 & & 85 & & & 25.6 \\
\hline \multirow[t]{5}{*}{29} & 15 & & & 26.4 & 24 & 15 & & & 27.2 \\
\hline & 30 & & & 26.8 & & 30 & & & 27.7 \\
\hline & 50 & & & 27.7 & & 50 & & & 28.5 \\
\hline & 70 & & & 29.0 & & 70 & & & 29.4 \\
\hline & 85 & & & 30.5 & & 85 & & & 30.4 \\
\hline \multirow[t]{5}{*}{32} & 15 & & & 28.8 & 28 & 15 & & & 30.0 \\
\hline & 30 & & & 29.6 & & 30 & & & 30.8 \\
\hline & 50 & & & 31.1 & & 50 & & & 32.1 \\
\hline & 70 & & & 33.4 & & 70 & & & 33.8 \\
\hline & 85 & & & 36.1 & & 85 & & & 35.4 \\
\hline
\end{tabular}

a) 由于气候室内采用围护结构, 且实验提前 $1 \mathrm{~h}$ 将气候室环境调至实验设计工况, 因此假定气候室内壁面温度近似等于空气温度, 下述分 析中不考虑辐射影响. 计算SET时, 假定气候室中平均辐射温度等于空气温度, 人体活动水平为静坐, 代谢率为 $1.1 \mathrm{met}\left(1 \mathrm{met}=58.2 \mathrm{~W} / \mathrm{m}^{2}\right)^{[27]}$, 计 算工具见http://comfort.cbe.berkeley.edu/

且湿度越大, 增加越显著, 表明SET可以较好地表征温 湿度综合作用，可用于后续不同实验工况下热舒适分 析和综合评价指标.

\section{4 问卷}

本实验问卷设置分为两部分. 其中, 问卷背景信息 包括受试者性别、年龄、身高、在重庆居住时间、平 时所处环境等，问卷主观调查主要包括受试者的热感 觉、湿度感觉、热舒适、湿度刺激感、出汗、可感空 气品质、环境期望、环境可接受度等, 参考ASHRAE 55 主观测量的标尺设置 ${ }^{[27]}$. 考虑到本文重点研究湿度 对人员湿度舒适性及可感空气品质的影响, 表3给出了 本文涉及的主观评价指标及标尺设置. 由于人员整体
和局部对温湿度的反应不同，问卷针对不同问题同时 设置了不同部位的整体和局部主观评价问题.

\section{5 实验流程}

为减小室外气候对人员热经历的影响, 实验分别 在重庆典型冬季(12 1月)和夏季(7 8月)开展. 由于每 一季节实验工况较多, 为避免受试者参与实验的疲劳

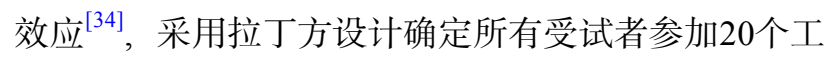
况实验的顺序. 实验前由测试人员提前 $1 \mathrm{~h}$ 将气候室热 环境参数调至设计工况(表2), 确保气候室内热环境的 均一稳定. 要求受试者提前 $30 \mathrm{~min}$ 到达气候室, 随后在 准备室更换服装, 并静坐一段时间, 以消除室外环境和 自身代谢影响. 期间由测试人员向受试者详细讲解问 
表 3 问卷主观评价指标及标尺

Table 3 Subjective indices and scales in questionnaire

\begin{tabular}{|c|c|c|c|c|c|c|c|}
\hline 标尺 & -3 & -2 & -1 & 0 & +1 & +2 & +3 \\
\hline 热感觉 ${ }^{a}$ & 冷 & 凉 & 稍凉 & 中性 & 稍暖 & 暖 & 热 \\
\hline 湿感觉 ${ }^{a)}$ & 非常干燥 & 干燥 & 稍干燥 & 中性 & 稍湿润 & 湿润 & 非常湿润 \\
\hline 感知空气品质 & 非常差 & 差 & 稍差 & 中性 & 还可以 & 好 & 非常好 \\
\hline 空气新鲜度 & 非常沉闷 & 沉闪 & 稍沉闪 & 中性 & 稍感清新 & 清新 & 非常清新 \\
\hline 身体刺激感 ${ }^{b)}$ & & & & 无刺激 & 有刺激, 但不明显 & 有明显刺激 & 强烈刺激 \\
\hline 温度/湿度/空气品质可接受度 & & & 完全不可接受 & $\begin{array}{c}+0 \text { 刚好可接受 } \\
-0 \text { 刚好不可接受 }\end{array}$ & 完全可接受 & & \\
\hline
\end{tabular}
激感.

a) 本研究主要分析整体热感觉和整体湿感觉, 不考虑局部; b) 本研究主要考虑空气温湿度对人体眼睛、鼻子、喉咙 3 个部位造成的刺

卷内容和实验流程, 并告知一些实验注意事项, 受试者 如有不适可随时终止实验.

冬夏季正式实验均为 $90 \mathrm{~min}$. 每次实验由两名受试 者参加, 实验在受试者进人气候室静坐后正式开始. 要 求受试者前 $30 \mathrm{~min}$ 每 $10 \mathrm{~min}$ 重复填写一次问卷, 后 $60 \mathrm{~min}$ 每 $15 \mathrm{~min}$ 重复填写一次问卷. 实验期间, 受试者 可以进行阅读、打字、听音乐等轻度活动, 避免大声 说笑、走动等剧烈活动. 同时, 采用德国美翠热舒适仪 (MI 6401, METREL)对室内的空气温度、相对湿度、 黑球温度、空气流速等物理参数进行测量(空气温度: $-20 \sim 60^{\circ} \mathrm{C}$, 精度 $\pm 0.20^{\circ} \mathrm{C}$; 相对湿度: $0 \sim 100 \%$, 精度 $\pm 2 \%$ ), 确保气候室的温度和相对湿度波动较小, 满足设计工 况的要求. 测试期间, 将仪器放置在距离受试者 $0.5 \mathrm{~m}$ 位置, 探头距离地面高度 $0.6 \mathrm{~m}$, 采样频率 $5 \mathrm{~min}$. 实验 结束后, 将连续测试数据导出到Excel保存.

\section{6 统计分析}

本文分析数据均为每个工况下 20 名受试者问卷投 票的均值. 首先, 通过重复测量方差分析确定受试者在 每个工况下主观投票稳定时间. 结果显示, 稳态环境下 受试者的主观感觉投票均在 $30 \mathrm{~min}$ 内达到稳定. 因此, 分析时采用每个工况下 60 90 min内 20 名受试者3次投 票的平均值作为该稳态工况下受试者投票均值(平均 值土标准差). 采用二维组间方差分析(two-way ANOVA) 分析不同温湿度水平下各主观测量指标的差异性, 采 用单因素方差分析(analysis of variance, ANOVA)分析 同一温度水平下各相对湿度水平引起的主观投票的差 异. 采用一元、二元线性回归和非线性回归对主观感 觉变量和温湿度等的相关关系进行回归分析. 所有统 计检验的显著性水平均取 $\alpha \leqslant 0.05$.

\section{2 结果与讨论}

\section{1 热反应}

\section{1 .1 湿感觉}

图2给出了冬夏季各个工况下 20 名受试者的稳态 湿感觉投票分布箱体图. 可以看出, 冬夏季受试者的湿 感觉投票值在偏凉、中性、偏暖环境下近似，均值均 在 0 上下，不同于热环境下投票值. 二维组间方差分析 结果显示, 冬夏季不同温度水平下受试者湿感觉投票 有显著差异 $(P<0.05)$, 不同湿度水平下投票值也有显著 性差异 $(P<0.05)$. 进一步采用单因素方差分析对同一温 度水平下不同湿度影响进行分析, 图2(a)显示夏季温度 为 23 和 $26^{\circ} \mathrm{C}$ 时, 各湿度水平下湿感觉无显著差异 $(P>0.05)$, 而 $29^{\circ} \mathrm{C}$ 时只有 $15 \%$ 和 $85 \%$ 相对湿度水平有显 著性差异 $(P<0.05)$. 这可能是由于这些工况接近中性热 环境, 湿度对人员主观感觉的影响不显著. 相比, 当温 度升高到 $32^{\circ} \mathrm{C}$ 时， 5 种湿度水平之间具有显著性差异 $(P<0.05)$, 人员的湿感觉随着相对湿度的增加而显著升

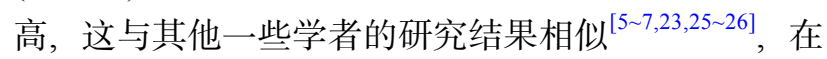
$32^{\circ} \mathrm{C} / 85 \% \mathrm{RH}$ 工况下, 人员的湿感觉甚至达 1.5 . 冬季不 同工况下受试者湿感觉变化趋势与夏季近似, $28^{\circ} \mathrm{C}$ 时 不同湿度水平下湿感觉存在显著差异 $(P<0.05)$. 这一结 果表明, 人们对相对湿度变化的可接受度要远高于对 温度变化的接受度, 中性及偏冷环境下较大的湿度变 化对人员湿感觉的影响不大, 但偏热环境下人员对湿 度变化较敏感.

\subsection{2 感知身体刺激感}

空气湿度不仅会影响人员角膜前泪腺膜水分蒸发, 引起眼睛刺激, 同时会影响空气焓值, 引起人体呼吸道 对流和蒸发散热变化, 对鼻子、喉咙等产生刺激 ${ }^{[17,19]}$. 
(a) 夏季

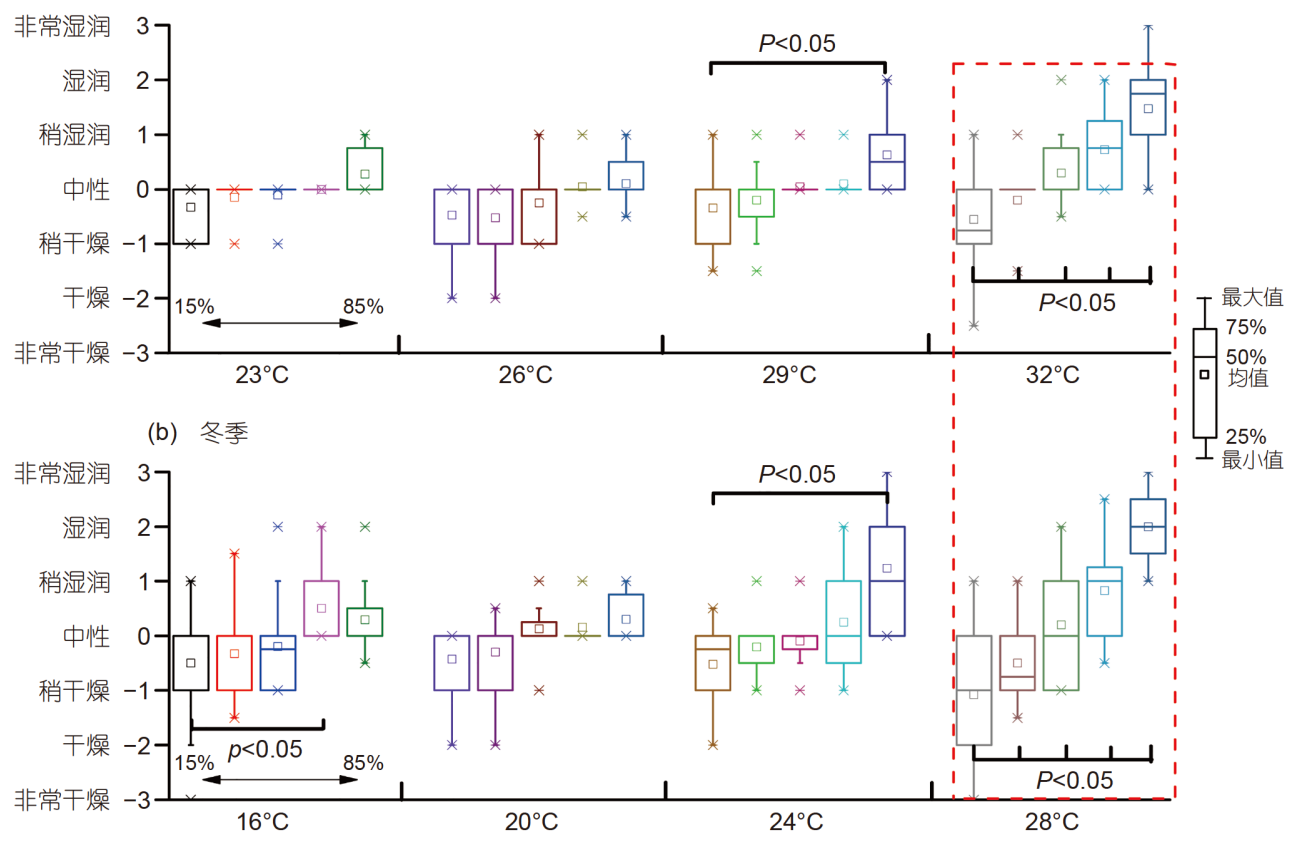

图 2 (网络版彩色)不同工况下受试者稳态湿感觉. (a) 夏季; (b) 冬季

Figure 2 (Color online) Mean humidity sensation of subjects under different conditions. (a) Summer; (b) winter

统计冬夏季不同工况下，实验过程中受试者对身体各 部位刺激感(眼睛、鼻子、喉咙)投票非 0 值(即表 3 中身 体刺激感投票为 $1,2,3)$ 的投票数占总投票数的百分比, 如图3所示. 为便于反映温湿度综合影响，图3以SET为 横坐标，4段折线分别表示冬夏季每一温度下相对湿度 从 $15 \%$ 逐渐增加到 $85 \%$ 引起的SET值的增加.

可以看出, 不同温度水平下, 受试者身体各部位刺

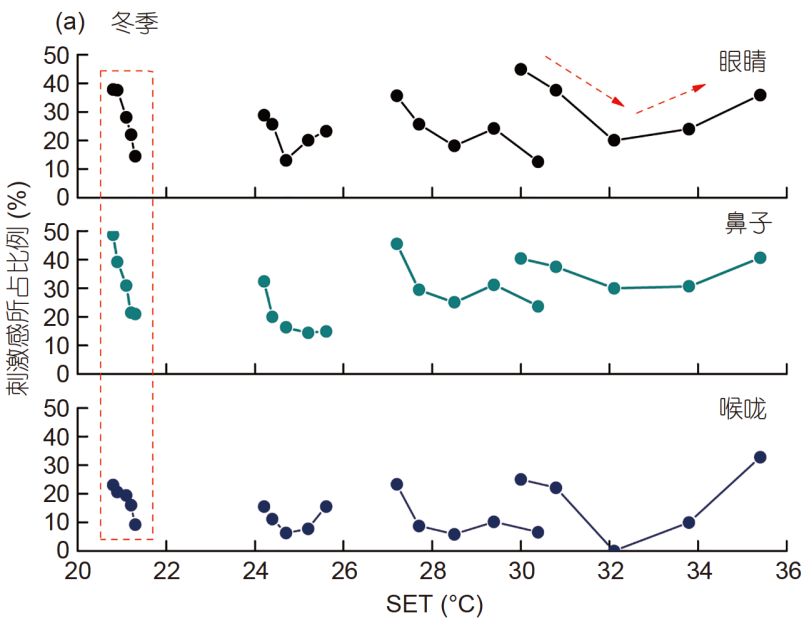

激感随湿度增加呈现不同的变化趋势. 图3(a)显示，在 $16^{\circ} \mathrm{C} / 15 \% \mathrm{RH}$ 工况下，受试者感受到眼睛、鼻子、喉咙 的刺激感比例都最大, 分别为 $37 \%, 48 \%, 23 \%$. 随着SET 值增大(湿度逐渐增加), 投票刺激感比例逐渐减小, 相 对湿度为 $85 \%$ 时达到最低，表明受试者在 $16^{\circ} \mathrm{C} / 85 \% \mathrm{RH}$ 时感受到环境的刺激感最小. 这可能是因为低温环境 下本身空气中含湿量较低，低相对湿度下空气含湿量

(b) 夏季

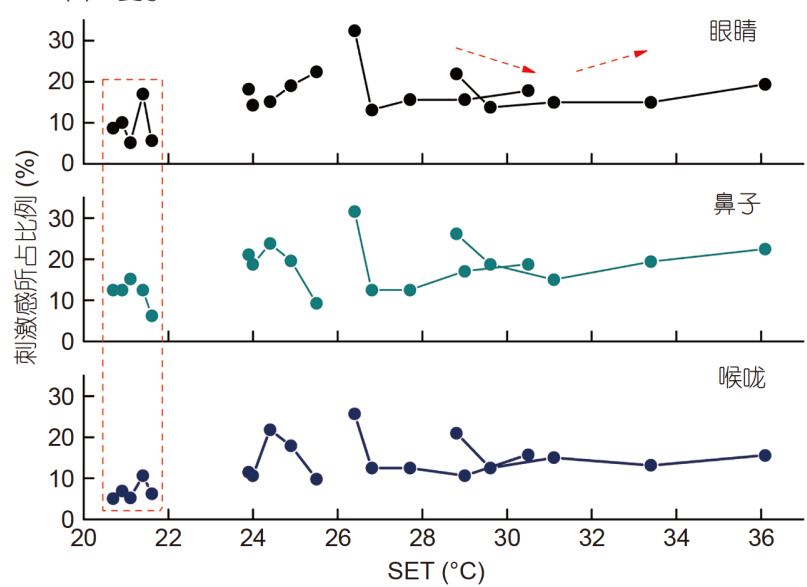

图 3 (网络版彩色) 冬夏季不同工况对受试者身体刺激感投票比例. (a) 冬季; (b) 夏季

Figure 3 (Color online) Stimuli sensation vote proportion of subjects under winter and summer conditions. (a) Winter; (b) summer 
更低，因而较多受试者感受到空气干燥带来的刺激感, 尤其是鼻子, 刺激感比例最大 $(48 \%)$. 相比之下, 当空气 温度为 20,24 和 $28^{\circ} \mathrm{C}$ 时, 受试者刺激感投票比例呈先降 低后增加的U型趋势. 例如, $28^{\circ} \mathrm{C} / 15 \%$ 相对湿度下各部 位刺激感比例分别为 $15 \%, 40 \%$ 和 $25 \%$, 而当湿度增加 到 $50 \%$ 时，其比例降低至最低，分别为 $20 \%, 30 \%$ 和 $0 \%$. 随着湿度继续增加，其刺激感比例又逐渐增加，到 $85 \%$ 时比例最大, 达 $35 \%, 40 \%$ 和 $32 \%$. 这可能是由于偏热环 境下高湿度抑制了人体黏膜水分蒸发和呼吸散热, 受 试者感到刺激的比例增加. 图3(b)显示，夏季受试者身 体各部位感知到的刺激感比例整体低于冬季. 这可能 是由于夏季温度增加, 即使相对湿度水平与冬季一样, 空气中绝对含湿量增加，对人员皮肤和呼吸道黏膜表 面的水分蒸发影响较小，人员感到刺激感的比例较低. 在 23 和 $26^{\circ} \mathrm{C}$ 时，受试者刺激感投票比例随着相对湿度 的增加变化不大，且在中性湿度 $50 \% \mathrm{RH}, 70 \% \mathrm{RH}$ 下比 例有一定波动. 温度为 29 和 $32^{\circ} \mathrm{C}$ 时, 其比例也呈近似 $\mathrm{U}$ 型变化，但相比图3(a)不十分显著，表明高湿和低湿一 定程度上会刺激人体, 引起局部不适, 但与环境温度存 在耦合作用.

\subsection{3 空气品质可接受度}

已有大量研究表明 ${ }^{[16,35,36]}$, 空气湿度会引起人员眼 睛不适、感官刺激、呼吸不适，以及室内潮湿、霉 菌、污染物挥发等环境问题，显著影响人员可感知空
气品质. 但由于人员感知有一定的适应性, 随着暴露时 间的延长，人员对湿度引起的空气品质感知的敏感性 也会逐渐下降. 图4对比了冬夏季不同工况下 20 名受试 者在暴露初始时刻 $(0 \mathrm{~min})$ 和结束时刻 $(90 \mathrm{~min})$ 的空气 品质可接受度的投票均值. 可以看出, 不同季节和温湿 度工况下，受试者对空气品质的可接受度随暴露时间 变化. 当相对湿度为 $15 \%$ 时, 不论是冬季还是夏季温度 工况，受试者对空气品质可接受度在实验初始阶段较 高, 而在暴露结束时明显降低, 表明长时间暴露在极低 湿度环境下, 人员的可感空气品质会降低. 这一现象在 冬季更明显，即使相对湿度增加到 $30 \%$ 和 $50 \%$, 受试者 对空气品质可接受度投票在实验结束时均不同程度地 降低. 在夏季时, 除了 $15 \% \mathrm{RH}$, 其余湿度水平下受试者 在暴露结束时可感空气品质均显著提高. 分析其原因, 可能是由于人员长时间暴露后产生了一定适应性 ${ }^{[2]}$, 降低了对湿度感知的敏感性，因此对空气品质的可接 受度提高.

\section{2 热环境评价}

\subsection{1 热感觉及空气新鲜度随呼吸热损失变化} 空气湿度一方面会通过影响人体皮肤表面的水分 蒸发而影响散热，另一方面也会通过影响呼吸道换热 间接影响人体对热环境和空气品质的感知. 低温低湿 的空气会增大人体呼吸系统的对流和蒸发冷却换热量,

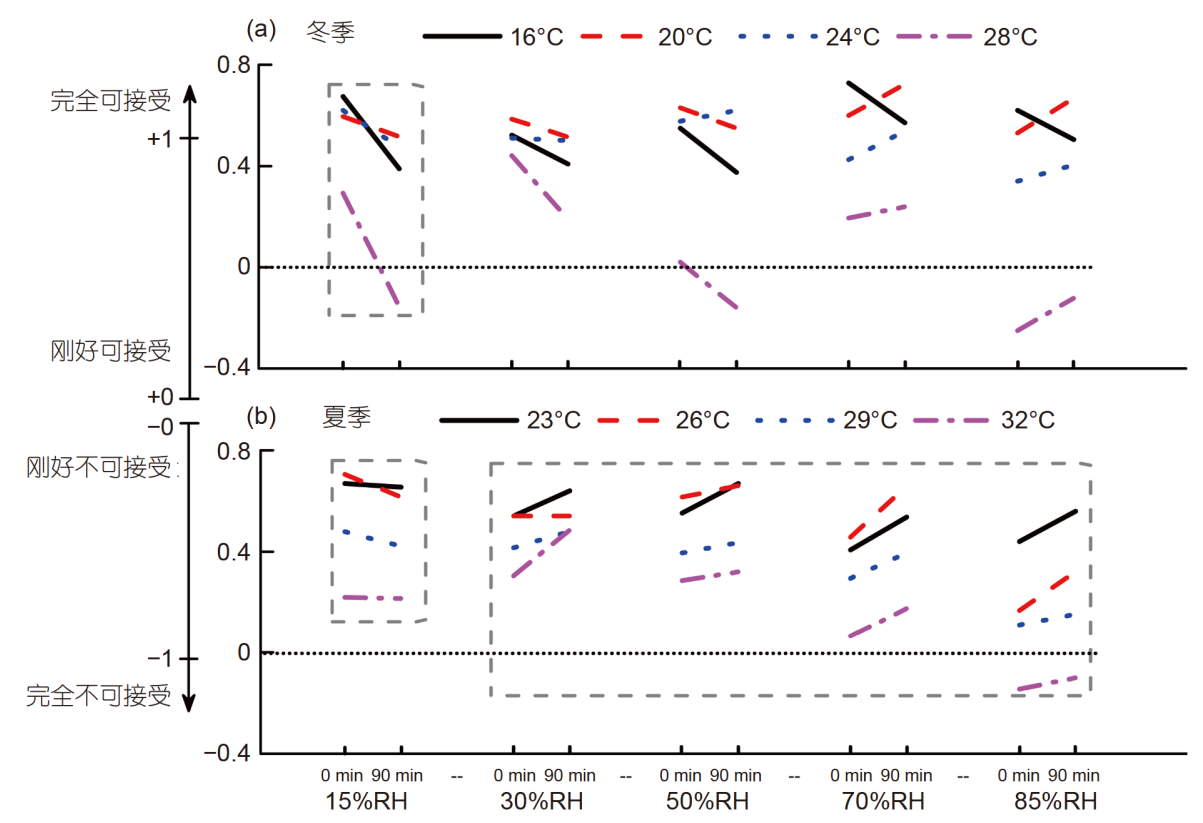

图 4 (网络版彩色)不同工况下受试者空气品质可接受度随时间变化. (a) 冬季; (b) 夏季

Figure 4 (Color online) Air quality acceptability of subjects changes with time under different conditions. (a) Winter; (b) summer 
而高温高湿的空气则会抑制呼吸道散热. 这里参考 ASHRAE Handbook ${ }^{[37]}$ 中人体呼吸道换热计算公式(式 (1)和(2)), 计算不同工况下受试者呼吸对流和蒸发换 热量.

$C_{\text {res }}=0.0014 M\left(34-t_{\mathrm{a}}\right)$,

$E_{\text {res }}=0.0173 M\left(5.87-P_{\mathrm{a}}\right)$,

式中, $C_{\mathrm{res}}$ 为呼吸对流热损失, $\mathrm{W} / \mathrm{m}^{2} ; E_{\mathrm{res}}$ 为呼吸蒸发热 损失, $\mathrm{W} / \mathrm{m}^{2} ; M$ 为代谢率, 本文假定代谢率为 $1.1 \operatorname{met}($ 静 坐活动), 折合 $65 \mathrm{~W} / \mathrm{m}^{2} ; t_{\mathrm{a}}$ 为环境温度, ${ }^{\circ} \mathrm{C} ; P_{\mathrm{a}}$ 为空气水 蒸气分压力, $\mathrm{kPa}$.

通过式(1)和(2), 可以得到冬夏季不同工况下受试 者总的呼吸热损失 $Q_{\mathrm{res}}\left(Q_{\mathrm{res}}=C_{\mathrm{res}}+E_{\mathrm{res}}\right)$. 图5给出了稳态 环境下受试者热感觉投票(thermal sensation vote, TSV) 和空气新鲜感投票(air freshness vote, AFV)随呼吸总热 损失 $\left(Q_{\mathrm{res}}\right)$ 的变化. 从图5(a) 可以看出, 不论是冬季还是 夏季, 受试者的热感觉均随呼吸总热损失的增加而降 低, 两者呈显著的线性负相关 $\left(R^{2}=0.77\right)$. 相比之下, 图 5 (b)中人员的感知空气新鲜度随呼吸总热损失的增加而 提高, 两者呈显著正相关 $\left(R^{2}=0.68\right)$. 对图 5 中的数据进 行线性拟合，可以得到人员的呼吸总热损失每增加 $1 \mathrm{~W} / \mathrm{m}^{2}$, 热感觉显著降低 0.66 个刻度, 而感知空气新鲜 度提高 0.22 个刻度. 这可能是由于提高呼吸道热损失增 加了呼吸道的冷却效应, 改善了人员对空气品质的感
知, 觉得空气更新鲜.

\subsection{2 热感觉及感知空气品质随SET变化}

图2 5分析表明，环境的温湿度会显著影响人员的 主观感觉评价, 且温湿度之间存在显著的耦合作用. 因 此，为了全面评价温湿度综合作用对人体热感觉和感 知空气品质的影响，图6给出了受试者稳态热感觉和感 知空气品质随SET变化. 可以看出, 不同工况下受试者 的热感觉和感知空气品质与SET之间存在显著的线性 关系. 冬季环境下(图6(a)), 随着 SET从 $20.8\left(16^{\circ} \mathrm{C} / 15 \%\right.$ $\mathrm{RH})$ 增加到 $35.4^{\circ} \mathrm{C}\left(28^{\circ} \mathrm{C} / 85 \% \mathrm{RH}\right)$, 热感觉也逐渐从-2.1 增加到 2.9. 同样地, 夏季环境下(图6(b)), 随着SET从 $20.7\left(23^{\circ} \mathrm{C} / 15 \% \mathrm{RH}\right)$ 增加到 $36.1^{\circ} \mathrm{C}\left(32^{\circ} \mathrm{C} / 85 \% \mathrm{RH}\right)$, 热感 觉也逐渐从- 0.9 增加到 2.9 , 进一步证实了 SET指标与 人员热感觉具有较好的线性关系 ${ }^{[6,25,38]}$. 相比之下, 人 员感知空气品质随SET的变化小于人员热感觉随SET 的变化. 冬季环境下随SET增加, 感知空气品质从 0.5 降 低到 -0.5 , 而夏季从 1.25 降低到 -1.1 , 表明人员对温湿度 变化引起的热感觉的敏感度大于室内空气品质.

式(3) (6)分别给出了冬季和夏季环境下人员 TSV, AQV随SET的线性回归模型. 不同模型的回归系数显 示，人员的TSV与SET正相关，而AQV与SET负相关, 且模型系数的绝对值的大小进一步证实了 TSV对SET 变化的敏感度要大于AQV对SET变化的敏感度.

冬季:
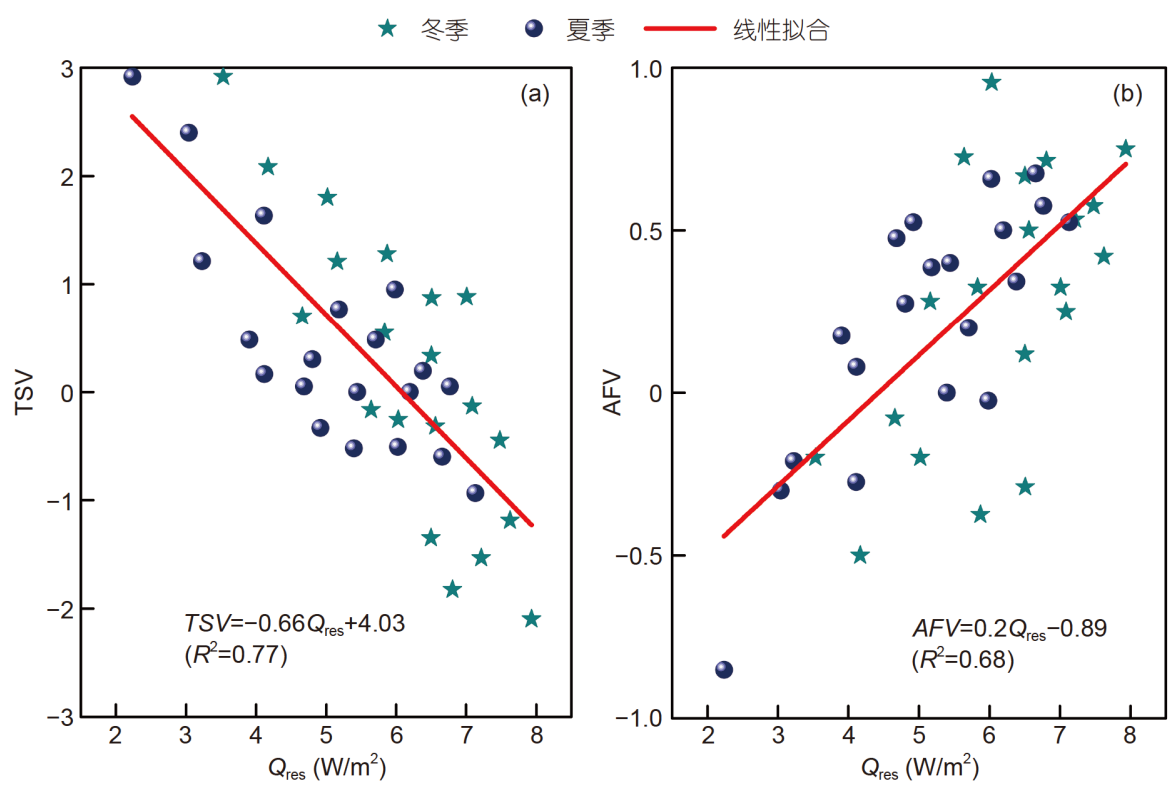

图 5 (网络版彩色)不同工况下热感觉和空气新鲜感随呼吸总热损失变化. (a) 热感觉; (b) 空气新鲜感

Figure 5 (Color online) Changes of TSV and AFV with $Q_{\text {res }}$ under different conditions. (a) TSV; (b) AFV 

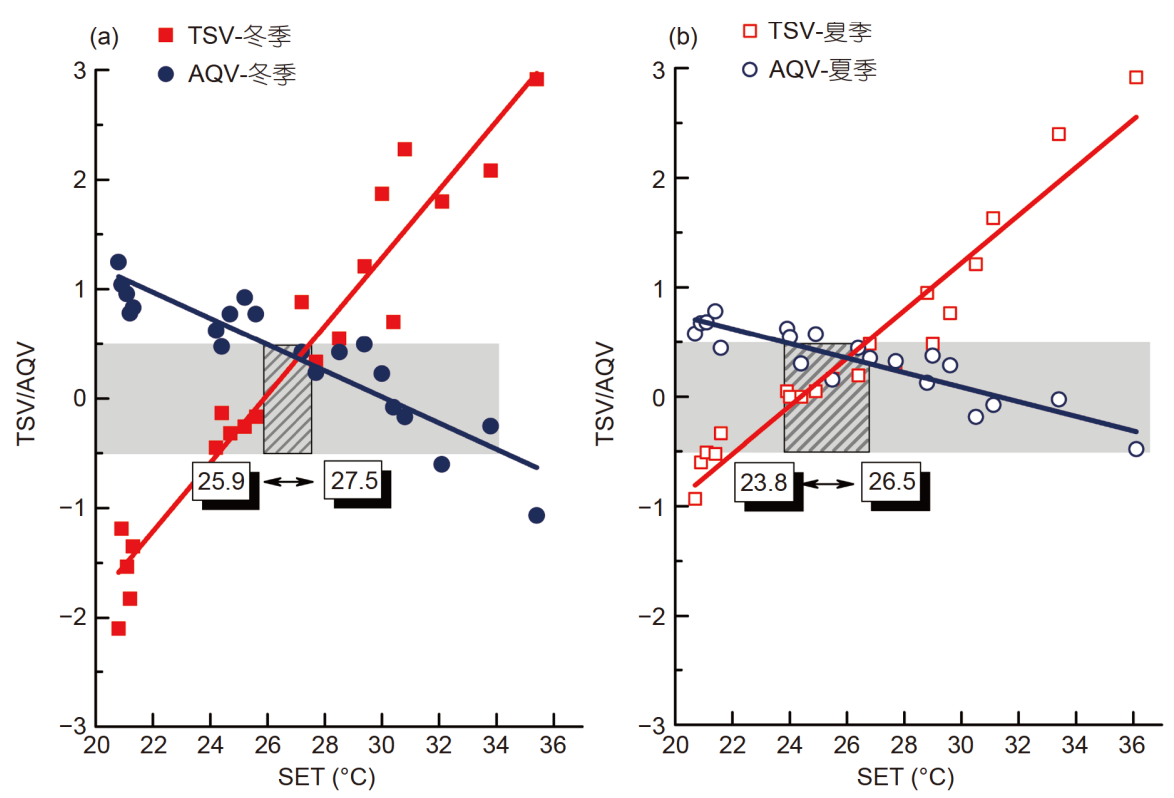

图 6 (网络版彩色)不同工况下热感觉和感知空气品质随SET变化. (a) 冬季; (b) 夏季

Figure 6 (Color online) Changes of TSV and AQV with SET under different conditions. (a) Winter; (b) summer

$T S V=0.31 S E T-8.08, \quad\left(R^{2}=0.94\right)$,

$A Q V=0.12 S E T+3.6, \quad\left(R^{2}=0.84\right)$,

夏季:

$$
\begin{array}{ll}
T S V=0.22 S E T-5.33, & \left(R^{2}=0.94\right), \\
A Q V=0.07 S E T+2.08, & \left(R^{2}=0.80\right) .
\end{array}
$$

\subsection{3 温湿度耦合作用下舒适区间}

虽然现有学者研究了相对湿度对人员热舒适的影 响，但一般多关注夏季偏热环境 ${ }^{[2 \sim 8]}$, 且实验样本量有 限, 较少对较大温度和湿度范围内的舒适评价开展研 究. 本研究实验结果证实了冬夏季空气湿度耦合温度 会对人体热舒适产生影响, 在室内热环境设计时不能 忽略. 对暖通从业者来讲, 他们更关注如何将湿度这一 影响量化来指导室内温度设计. 对于人工供冷供暖环 境, 国际标准ASHRAE $55^{[27]}$ 以人员预测热感觉投票 (predicted mean vote, $\mathrm{PMV}) \in(-0.5,+0.5)$ 为限值, 给出 了 $90 \%$ 人员可接受的温湿度舒适区间. 图6给出了热感 觉随SET变化的回归模型, 在此基础上参考ASHRAE 55 中舒适区间定义, 以人员实际热感觉投票 $T S V \in(-$ $0.5,+0.5)$ 为参考, 则可计算冬夏季满足热舒适要求的 对应SET值. 根据SET定义，等SET下不同热环境参数 变化对应的人员热感觉相同 ${ }^{[33]}$, 因此可以计算在保证 人员同样热舒适情况下不同温度对应的相对湿度变化. 此外, 考虑到一般建筑中人员对热环境的可接受度更
高，本文同时计算了满足 $80 \%$ 人员满意率，即TSVE($0.75,+0.75)$ 条件下对应的SET值.

基于实验得到的回归模型(式(3)和(5))和计算得到 的SET值，同时参考实验设置，假定平均辐射温度等于 空气温度，平均风速 $0.1 \mathrm{~m} / \mathrm{s}$ ，人员服装热阻夏季 0.4 clo、冬季 $1.2 \mathrm{clo}$, 代谢 $1.1 \mathrm{met}$, 则可以得到在等SET条 件下温度和湿度的耦合变化关系. 结合室内热环境营 造中空气相对湿度变化范围为 $30 \% \sim 70 \%{ }^{[39]}$, 分别以冬 夏季等SET值为约束条件, 计算在等SET条件下不同温 度水平对应的适宜的相对湿度范围, 如图7所示. 图7中 不同形式阴影分别表示冬季和夏季 $80 \%$ 和 $90 \%$ 人员满 意率对应的温度-相对湿度变化范围. 图7同时标注了不 同温湿度区间对应的等SET 左右边界线. 可以看出, 冬 季满足人员 $80 \%$ 和 $90 \%$ 舒适区间对应的SET值分别为 $24.2 \sim 27.5^{\circ} \mathrm{C}, 23.6 \sim 28.5^{\circ} \mathrm{C}$, 夏季对应的SET值分别为 $22 \sim 26.5^{\circ} \mathrm{C}, 20.8 \sim 27.6^{\circ} \mathrm{C}$. 此外, 图7显示, 随着相对湿度 增加, 在满足等SET条件下, 人员可接受温度逐渐降低, 冬季相对应的温度区间为 $18.6(70 \%) \sim 23.4^{\circ} \mathrm{C}(30 \%)$, 夏 季为 $22.0(70 \%) \sim 29.5^{\circ} \mathrm{C}(30 \%)$, 量化了湿度耦合温度对 人员热感觉的影响.

\section{3 讨论}

\subsubsection{ASHRAE 55温湿度区间对比}

将本研究得到的冬夏季温湿度区间与现有ASH- 


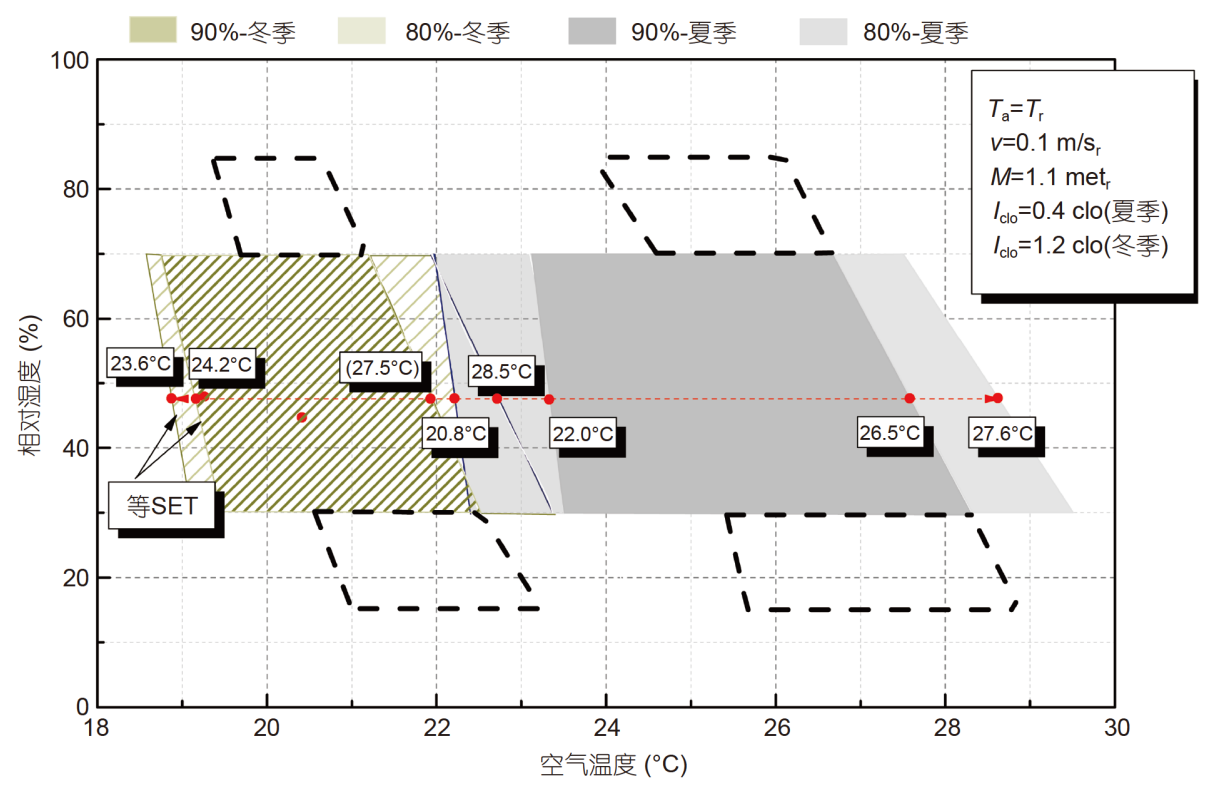

图 7 (网络版彩色) 等SET条件下可接受的温度-相对湿度区间

Figure 7 (Color online) Acceptable temperature-relative humidity range with equivalent SET values

RAE 55标准给出的冬夏季舒适温度范围(冬季：20 $24^{\circ} \mathrm{C}$ (服装热阻 $1.0 \mathrm{clo}$ ), 夏季: $23.5 \sim 27^{\circ} \mathrm{C}$ (服装热阻 0.5 clo))进行比较, 本研究得到的人员舒适温度上下限要 高于ASHRAE 55标准推荐值. 分析其原因, 一方面, 由 于本实验计算的舒适区间的人员服装热阻和ASHRAE 55中给定的冬夏季标准服装热阻有一定差异, 实验中 冬季服装热阻偏高, 起到保温作用, 夏季服装热阻偏 低, 促进人体散热, 因而获得的人员舒适温度上下限范 围更广. 另一方面, 本实验基于中国人群，而ASHRAE 55 标准基于欧美人群，不同地区人群的热适应性也可 能引起舒适区间的差异. 由于重庆全年高湿气候特点, 实验中受试者对高湿度有一定的适应性，扩宽了可接 受温度范围 ${ }^{[40]}$. 此外, ASHRAE 55 新版标准并没有给 定相对湿度下限，而相对湿度上限则考虑含湿量不高 于 $12 \mathrm{~g} / \mathrm{kg}^{[27]}$, 这一限值并不方便实际建筑中室内空气 温度和湿度的协调调控. 相比之下, 本研究基于中国人 群和大样本实验室测试得到的温度-相对湿度区间既 保证了人员的热舒适度要求, 又可以较好地为我国室 内热环境设计和营造提供一定的参考.

\subsection{2 极端温湿度环境对人员热舒适影响}

上述实验结果显示, 环境温湿度对人员的热舒适 和感知空气品质都存在显著影响. 由于人员的可感知 空气品质与空气焓值呈较好的线性负相关关系 ${ }^{[17,19]}$, Toftum和Fanger ${ }^{[41]}$ 曾建议采用人的呼吸系统对湿度的
感觉来定义热湿舒适区的湿度影响. 本文研究结果也 证实, 在低温和和高温情况下, 空气湿度的变化显著影 响人员热舒适和对空气品质的评价, 因此室内热环境 舒适区间的确定也应考虑这两方面的综合影响. 采用 同样的计算方法，图7绘制了舒适区间外相对湿度低于 $30 \%$ 和高于 $70 \%$, 同时满足热感觉 TSV $\in(-0.5,0.5)$ 和感 知空气品质 $\mathrm{AQV} \in(-0.5,05)$ 的温湿度区间. 考虑到极端 环境下人员对热舒适的要求更严格, 这里仅给出了满 足 $90 \%$ 人员满意度的冬夏季温度-相对湿度区间. 可以 看出, 极端环境下由于空气湿度对人员热舒适影响显 著, 人员可接受温度-相对湿度区间更窄.

分析其原因, 偏热环境下相对湿度增加, 一方面抑 制了皮肤表面的汗液蒸发带走热量, 降低了人体皮肤 表面蒸发热损失 ${ }^{[6,7]}$, 另一方面高温高湿情况下空气含 湿量大, 湿润的空气抑制了呼吸热损失, 使人员感知的 空气新鲜度降低(图5), 导致随着相对湿度增加, 人员可 接受温度上下限降低. 这一现象在高湿度情况下更显 著. 图7显示, 湿度大于 $70 \%$ 后人员可接受温度的降低 速率要高于等SET条件下低湿环境. 同样地, 低湿环境 下空气湿度对人体热舒适的影响也是两方面的. 一方 面, 随着温度降低, 即使相对湿度相等, 空气中实际含 湿量降低 ${ }^{[25]}$, 因而干燥的空气会引起身体的刺激感. 图 3 显示, $16^{\circ} \mathrm{C}$ 时, 随着相对湿度降低, 人员身体各部位刺 激感投票的比例显著增加. 低温低湿的空气同时也会 
刺激呼吸系统和眼睛泪膜，造成鼻腔、眼睛和呼吸道 黏膜的水分减少 ${ }^{[17]}$, 引起不适, 降低人员的可接受温 度上下限. 另一方面, 低温环境下, 温度不变、相对湿 度降低时, 总的蒸发热损失会增加, 附加的蒸发热损失 减少了干热损失, 导致皮肤表面温度降低, 因而人员在 低湿度时感觉更冷 ${ }^{[42]}$. 在这种情况下, 相对湿度的增加 可以补偿人体的热损失，图7中人员可接受温度限值 降低.

需要注意的是，冬季情况下由于服装吸湿放湿作 用, 相对湿度对人员热舒适的影响更加复杂. 一些学者 研究了服装的“冷却效应(after chill), ${ }^{\text {[4 [3] }}$, 但研究多基于 夏季偏热、人体出汗情况，服装的吸湿和放湿强化了 人体蒸发散热, 起到积极作用. 然而, 冬季冷环境下, 人体无显性出汗蒸发，这时如果空气湿度过高(比如本 研究RH $>70 \%$ ), 人体服装会从空气中吸收多余水分, 导 致服装材料的导热特性发生变化, 热阻降低, 湿阻增加. 加上冬季皮肤表面的温度远高于服装外表面空气温度， 两者间的较大温差导致储存在服装中的一部分水分不 断汽化蒸发, 不断从人体吸收热量. 这种情况下, 高空 气湿度反而会引起人体的冷不适 ${ }^{[42]}$, 这也部分揭示了 南方地区冬季人员感觉“更冷”的原因．本研究冬季实 验时由于暴露时间有限，暂时没有考虑服装吸湿效应 的影响，建议后期关于温湿度对人员热舒适影响的研 究，也应充分考虑低温和高温环境下服装微环境的综 合影响, 分情况进行评价. 对于室内热环境的营造, 也 应考虑不同温度背景下相对湿度的积极或者消极影响, 保证室内热环境的舒适健康营造.

\section{3 结论}

通过人工气候室冬夏季大样本人体热舒适实验, 分析了不同温湿度耦合作用下的人员热反应，得到的 主要结论如下:

(1) 相对湿度对人员湿感觉的影响在偏热环境下显 著 $\left(\right.$ 夏季 $32^{\circ} \mathrm{C}$, 冬季 $28^{\circ} \mathrm{C}$ ), 湿感觉随相对湿度增加而显 著增加. 冬季低温环境下, 由于空气含湿量较低, 人员感 知身体各部位的刺激感显著, 尤其是鼻子, 随着温湿度 增加, 刺激感比例呈先降低后增加趋势. 长时间暴露在 极低湿度环境下，人员的可感空气品质降低, 而高湿环 境下人员对湿度变化敏感性降低, 可感空气品质提高.

(2) 人员热感觉和感知空气新鲜度与呼吸总散热 损失呈显著线性相关关系. 随呼吸总热损失增加, 人员 热感觉降低, 感知空气新鲜度增加, 且对热感觉影响(回 归系数：-0.66)比对感知空气新鲜度影响(回归系数: 0.2 )更显著.

(3) 冬夏季环境下人员热感觉和感知空气品质与 SET呈不同线性关系. SET每增加 $1^{\circ} \mathrm{C}$, 对热感觉的影响 显著大于对可感空气品质的影响，表明人员对相对湿 度变化的可接受度要远高于对温度变化的可接受度.

(4) 基于 TSV $\in(-0.5,+0.5)$ 和 $\mathrm{TSV} \in(-0.75,+0.75)$, 得到了冬夏季在满足 $90 \%$ 和 $80 \%$ 人员满意率、等 $\mathrm{SET}$ 限值条件下, 人员可接受的温度-相对湿度舒适区间. 在 等SET条件下, 人员可接受的温度上下限随相对湿度增 加逐渐降低，且大于国际标准ASHRAE55推荐的温度 上下限, 量化了湿度耦合温度对人员热舒适的影响, 可 为室内热环境设计和营造提供一定参考.

\section{参考文献}

1 Nagda N L, Rector H E. A critical review of reported air concentrations of organic compounds in aircraft cabins. Indoor Air, 2003, 13: 292-301

2 Givoni B, Khedari J, Wong N H, et al. Thermal sensation responses in hot, humid climates: Effects of humidity. Build Res Inf, 2006, 34: 496-506

3 Fanger P O. Thermal Comfort-Analysis and Application in Environment Engineering. Copenhagen: Danish Technology Press, 1970

4 Mcintyre D A. Indoor Climate. London: Applied Science Publishers, 1980

5 McNall P E, Jaax F H, Rohles R G, et al. Thermal comfort (thermally neutral) conditions for three levels of activity. ASHRAE Trans, 1967, 73: 1-3

6 Tan M L. Effect of air humidity and velocity on human thermal sensation in summer (in Chinese). Doctor Dissertation. Chongqing: Chongqing University, 2012 [谈美兰. 夏季相对湿度和风速对人体热感觉的影响研究. 博士学位论文. 重庆: 重庆大学, 2012]

7 Jing S, Li B, Tan M, et al. Impact of relative humidity on thermal comfort in a warm environment. Indoor Built Environ, 2013, 22: 598-607

8 Vellei M, Herrera M, Fosas D, et al. The influence of relative humidity on adaptive thermal comfort. Build Environ, 2017, 124: 171-185

9 Hänninen O O. WHO guidelines for indoor air quality: Dampness and mold. In: Adan O C G, Samson RA, eds. Fundamentals of Mold Growth in Indoor Environments and Strategies for Healthy Living. Wageningen: Wageningen Academic Publishers, 2011

10 World Health Organization (WHO). Development of WHO guidelines for indoor air quality: Dampness and mould. Report of a Working Group Meeting. Bonn, Germany, 2007, 17-18/10

11 Kennedy K, Grimes C. Indoor water and dampness and the health effects on children: A review. Curr Allergy Asthma Rep, 2013, 13: 672-680 
12 Mendell M J, Mirer A G, Cheung K, et al. Respiratory and allergic health effects of dampness, mold, and dampness-related agents: A review of the epidemiologic evidence. Environ Health Perspect, 2011, 119: 748-756

13 Tischer C G, Hohmann C, Thiering E, et al. Meta-analysis of mould and dampness exposure on asthma and allergy in eight European birth cohorts: An ENRIECO initiative. Allergy, 2011, 66: 1570-1579

14 Zhang Y P, Li B Z, Huang C, et al. Ten cities cross-sectional questionnaire survey of children asthma and other allergies in China (in Chinese). Chin Sci Bull, 2013, 58: 2504-2512 [张寅平, 李百战, 黄晨, 等. 中国10城市儿童哮喘及其他过敏性疾病现状调查. 科学通报, 2013, 58: 25042512]

15 Wang H, Li B Z, Yang Q, et al. Dampness in dwellings and its associations with asthma and allergies among children in Chongqing: A crosssectional study (in Chinese). Chin Sci Bull, 2013, 58: 2584-2591 [王晗, 李百战, 阳琴, 等. 重庆地区住宅室内潮湿问题和儿童哮喘及过敏性疾 病的相关性分析: 横断面调查. 科学通报, 2013, 58: 2584-2591]

16 Wei W, Ramalho O, Derbez M, et al. Applicability and relevance of six indoor air quality indexes. Build Environ, 2016, 109: 42-49

17 Toftum J, Jørgensen A S, Fanger P O. Upper limits of air humidity for preventing warm respiratory discomfort. Energy Build, 1998, 28: 15-23

18 Fang L, Clausen G, Fanger P O. Temperature and humidity: Important factors for perception of air quality and for ventilation requirements. ASHRAE Trans, 2000, 106: 503-510

19 Fang L, Wyon D P, Clausen G, et al. Impact of indoor air temperature and humidity in an office on perceived air quality, SBS symptoms and performance. Indoor Air, 2004, 14: 74-81

20 Wolkoff P, Nøjgaard J K, Troiano P, et al. Eye complaints in the office environment: Precorneal tear film integrity influenced by eye blinking efficiency. Occupat Environ Med, 2005, 62: 4-12

21 Fanger P O. Indoor air quality in the 21st century: Search for excellence. Indoor Air, 2000, 10: 68-73

22 Wolkoff P, Kjaergaard S K. The dichotomy of relative humidity on indoor air quality. Environ Int, 2007, 33: 850-857

23 Nevins R, Rohles F, Springer W, et al. Temperature-humidity chart for thermal comfort of seated persons. ASHRAE Trans, 1996, 72: 283-291

24 Atmaca I, Yigit A. Predicting the effect of relative humidity on skin temperature and skin wettedness. J Therm Biol, 2006, 31: 442-452

$25 \mathrm{Li} \mathrm{C}$, Liu H, Li B Z, et al. Seasonal effect of humidity on human comfort in a hot summer/cold winter zone in China. Indoor Built Environ, 2018, 28: $1-14$

26 Toftum J, Jørgensen A S, Fanger P O. Upper limits for indoor air humidity to avoid uncomfortably humid skin. Energy Build, 1998, 28: 1-13

27 American Society of Heating, Refrigerating and Air-Conditioning Engineers. Thermal Environmental Conditions for Human Occupancy. ASHRAE 55-2017. Atlanta, GA, 2017

28 International Standardization Organization. ISO 7730. Ergonomics of the Thermal Environment-Analytical Determination and Interpretation of Thermal Comfort Using Calculation of the PMV and PPD Indices and Local Thermal Comfort Criteria. Genevan, 2005

29 American Society of Heating, Refrigerating and Air-Conditioning Engineers. Ventilation for Acceptable Indoor Air Quality. ASHRAE 62.1-2013. Atlanta, GA, 2013

30 International Standardization Organization. EN 15251-2007. Indoor Environmental Input Parameters for Design and Assessment of Energy Performance of Buildings Addressing Indoor Air Quality, Thermal Environment, Lighting and Acoustics, 2007

31 Chinese Society of Architecture. Assessment Standard for Healthy Building (in Chinese). T/ASC 02-2016. Beijing, 2016 [中国建筑学会. 健康建 筑评价标准. T/ASC 02-2016. 北京, 2016]

32 Dj H, Ming C C, Kumaresan S. Effect of humidity on thermal comfort in the humid tropics. J Build Constr Plan Res, 2014, 2: 109-117

33 Gagge A P, Fobelets A P, Berglund L. A standard predictive index of human response to the thermal environment. ASHRAE Trans, 1986, 92: 709731

34 Lan L. Mechanism and evaluation of the effects of indoor environment quality on human productivity (in Chinese). Doctor Dissertation. Shanghai: Shanghai Jiao Tong University, 2010 [兰丽. 室内环境对人员工作效率影响机理及评价研究. 博士学位论文. 上海: 上海交通大学, 2010]

35 Broderick Á, Byrne M, Armstrong S, et al. A pre and post evaluation of indoor air quality, ventilation, and thermal comfort in retrofitted cooperative social housing. Build Environ, 2017, 122: 126-133

36 Wargocki P, Wyon D P. Ten questions concerning thermal and indoor air quality effects on the performance of office work and schoolwork. Build Environ, 2017, 112: 359-366

37 ASHRAE Technical Committees. ASHRAE Handbook-2013. Foundamentals-Chapter 9 Thermal Comfort, 2013 
38 Tian Y Y. Study of human thermal response in hot-humid environment (in Chinese). Doctor Dissertation. Beijing: Tsinghua University, 2001 [田元 媛. 热湿环境下人体热反应的研究. 博士学位论文. 北京: 清华大学, 2001]

39 Ministry of Housing and Urban-Rural Construction of China. Design Code for Heating Ventilation and Air Conditioning of Civil Buildings (in Chinese), GB 50736-2012. 2012 [中国住房和城乡建设部. 民用建筑供暖通风与空气调节设计规范, GB 50736-2012. 2012]

40 Li B, Du C, Tan M, et al. A modified method of evaluating the impact of air humidity on human acceptable air temperatures in hot-humid environments. Energy Build, 2018, 158: 393-405

41 Toftum J, Fanger P O. Air humidity requirements for human comfort. ASHRAE Trans, 1999, 2: 641-652

$42 \mathrm{Du} \mathrm{C}, \mathrm{Li} \mathrm{B}, \mathrm{Yu} \mathrm{W}$, et al. Moisture in clothing and its transient influence on human thermal responses through clothing microenvironment in cold environments in winter. Build Environ, 2019, 150: 1-12

43 Vivekanandan M V, Sreenivasan S. Dynamic transportation of water vapor through cotton and polyester-cotton blended fabrics: Part I: Indices characterizing moisture buffering and their interrelationships. J Eng Fiber Fabr, 2012, 7: 70-80 


\title{
Effect of air humidity on human acceptable thermal environments and evaluation
}

\author{
Chenqiu Du, Baizhan Li ${ }^{*}$, Hong Liu \& Chao Li \\ Joint International Research Laboratory of Green Buildings and Built Environments, Ministry of Education, Chongqing University, Chongqing 400045, \\ China \\ * Corresponding author, E-mail: baizhanli@cqu.edu.cn
}

Air humidity, coupled with temperature, has been verified with significant effect on human thermal comfort, specifically in warm environments. However, it lacks a holistic understanding on their comprehensive impacts in a wide range of relative humidity, especially in winter. This study aims to evaluate human thermal responses to air humidity, under a wide temperature range, and improve understandings of the role of air humidity on human comfort, with considering the effects of air humidity on human respiratory system. The work is expected to benefit to indoor thermal environment design and building energy saving. To this end, the study conducted a human exposure experiment in a well-controlled climate chamber in summer and winter respectively, with a combination of 20 temperature and relative humidity conditions (temperature: $16 / 20 / 24 / 28^{\circ} \mathrm{C}$ (winter), $23 / 26 / 29 / 32^{\circ} \mathrm{C}$ (summer), relative humidity: $15 \% / 30 \% / 50 \% / 70 \% / 85 \%$ ). Subjective evaluations of 20 subjects in each experimental condition were measured and total 800 samples were collected for analysis. Two-way ANOVA and ANOVA were conducted to test the significance of temperature and relative humidity on thermal perception evaluations of subjects. The results showed that the air humidity had neglectable effects on human humidity sensation in neutral and slightly warm environments; while significant increases of humidity sensation votes were found under warm conditions (summer: $32^{\circ} \mathrm{C}$; winter: $28^{\circ} \mathrm{C}$ ). With long term exposure, low relative humidity had remarkable stimulus on human body in cold environments, due to lower moisture content in air enhancing the evaporation on skin surface and mucosa; while the acceptability for air quality of subjects increased with increasing relative humidity under hot environment, which was attributed to long time thermal adaptation and low sensitivity to humidity changes. The heat loss from human respiration was calculated and related to human thermal sensation (TSV) and air freshness sensation (AFV): With increasing respiratory heat loss per $1 \mathrm{~W} / \mathrm{m}^{2}$, TSV decreased by 0.66 scale and AFV increased by 0.22 scale. To quantify such effects, the standard effective temperature (SET) was adopted as independent variable and the linear regressions between TSV and air quality sensation (AQV) and SET were built. Accordingly, taking TSV $\in(-0.5,+0.5)(90 \%$ people satisfaction) and $(-0.75,+0.75)(80 \%$ people satisfaction) as baselines, the upper and lower limits of SET for summer and winter were obtained. Combined with experimental conditions, the corresponding acceptable temperaturerelative humidity ranges regarding to equivalent SET were determined, with the same thermal comfort. The obtained temperature ranges were wider than that of ASHRAE 55 standards, which were inferred to differences of clothing insulation and thermal adaptation for different populations. Further, the results indicated that the extreme low and high air humidity affected the heat loss at skin surface and evaporative heat loss from respiratory, reducing the acceptable temperature limits. Overall, the work quantifies the coupling effect of air temperature and humidity on human thermal comfort; the comfort zones enable to obtain the range of relative humidity with given temperatures, and the range of temperature with given relative humidity, which is beneficial for designers and operators for building indoor thermal environments. Further work is discussed regarding to the microenvironment in clothes, especially for the negative effects of clothing absorption, which is worthy of consideration for heating design in cold-humid environments like hot summer and cold winter zone in China, to ensure human comfort and health.

air temperature, relative humidity, thermal comfort, air quality sensation, temperature-relative humidity range doi: 10.1360/TB-2019-0461 